\title{
A Method for Footprint Range Image Segmentation and Description
}

\author{
Yihong Ding, Xijian Ping, Min Hu, and Tao Zhang \\ Zhengzhou Information Science and Technology Institute, \\ Zhengzhou, Henan, China, 450002 \\ dingyihong@126.com
}

\begin{abstract}
In this paper, we firstly present a novel footprint range image segmentation method using the principal curvatures and the principal directions. Utilizing the principal curvatures information, we detect the peak areas as the seeds, and apply region growing to locate the edges of each patch. We apply the edge detection technology to the region growth rules, so the boundary localization is precise. To obtain more stable edge information, a multi-scale fusion approach is proposed to integrate the segmentation results calculated at different fitting sizes. After the segmentation, according to the shape characteristics of footprint, we use superquadric and saddle models to describe shape features of each patch. The experiments results on footprint range images show that the segmented patches and the descriptions represent footprint biometric information effectively and set a reliable basis for the further recognition.
\end{abstract}

\section{Introduction}

Biometric technologies are automated methods of recognizing a person based on physiological or behavioral characteristics. In the recent years, as the development of the information and automation technologies, biometrics is becoming the foundation of an extensive array of highly secure identification and personal verification solutions. Many features have been widely studied such as face, fingerprints, hand geometry, handwriting, iris, retinal, vein, and voice [1].

Determined by the personal characteristics of stature, skelecton and gait etc, footprint is a kind of singular and stable biometric feature and has been used in criminal detection by artificially recognition for many years [2]. But it is paid less attention in personal identification because the measure of footprint is easy to be interfered by ground conditions and personal behaviors. These interferences bring great difficulties for automatic data collection and analysis.

According to foot's dress, footprint can be classified into bare footprint, shoe footprint, sock footprint etc. Based on the imprint face, it also can be classified into stereo footprint, for example step in the mud or sand, and plane footprint such as step on the floor [2]. The bare footprint and the stereo footprint contains more physiological and behavioral characteristics, because the bare footprint reflects foot impress more directly than shoe and sock footprint and the stereo footprint holds 3Dimensional range information but the plane footprint shows only 2-Dimensional 
information. At the same time, the researches on the bare and stereo footprint show more challenges and difficulties. In the past years, some researches have been done in the field of footprint recognition. For instance, Tian et al have proposed a heavy impress slice based method to recognize the 3-Dimensional shoe footprint [3]. Nakajima and his colleagues have developed a personal recognition method by analyzing the gait information from a pair of bare footprints [4]. Kennedy et al have studied on making measurements on the inked barefoot impressions on paper [5].

We take the stereo bare footprint as our research object. The stereo bare footprint is obtained by stepping on the soft impressed face of great plasticity with barefoot. To extract the shape and range information of the footprint, the plaster cast of the footprint is produced by the processes of perfusing, molding and airing. Then a special distance measuring instrument is used to measure the range data of the footprint surface from the plaster cast. In this way, the bare stereo footprint can be described with discrete range data matrix.

In this paper, we propose a novel algorithm to segment the range image of the stereo bear footprint based on principal curvatures and principal directions information. And then a new method is put forward to descript the footprint. Section 2 presents our segmentation algorithm for footprint surface. Section 3 shows the footprint description method using the superquadric and saddle models. And section 4 gives the experiment results and the conclusion.

\section{Footprint Range Image Segmentation}

The technologies of range image segmentation have been extensively studied and can be roughly classified into three categories: edge based [6][7][8], region based [9][10] and hybrid techniques [11][12].

The segmentation characteristics of commonly used can derive through the principal curvature and the principal direction (for example: the normal vector is two principal directions cross products, the Gaussian curvature is the product of two principal curvatures, the mean curvature is the mean value of two principal curvatures). We may know by the differential geometry theory, two principal curvatures and its the principal direction had reflected completely the curved surface bending strain and the change tendency, and it is important basis of the range image segmentation. Many segmentation algorithms [9][10][11][12] often neglect the direction that has reflected the change tendency of the curved surface. Therefore the principal directions are important to the determination curved surface patch boundary position and the boundary direction.

In this section, we propose a region-growing algorithm based on principal curvatures information and principal directions information, which is important and often neglect by many segmentation algorithm. We apply the edge detection technology to the region growth rules, so the region-based approach can obtain precise boundary localization. According to the practical requirements of footprint analysis, our method stresses on extracting convex local surfaces. Our approach consists of three steps as follows: 1) preprocessing and pre-segmenting, 2) region growing based on the principal curvatures and their directions, 3) fusing segmentation results at different scales to reduce over-segmentation. 


\subsection{Preprocessing and Pre-segmenting}

The range image of the stereo bare footprint in our paper is obtained by measuring the discrete range data of the footprint plaster cast surface with an automated instrument. The collected range data can be digitalized and sent to a computer and saved as a range image. This kind of footprint data collection method has the advantage of cheap cost. But the collected foot range image has a large amount of noise that is caused by the complex ground condition and the quality of manufacturing the plaster cast. We firstly remove the impulsive noise with a $3 \times 3$ median filter. Then a Gaussian filter is used to smooth the image.

Limited by the precision of the collection instrument, the minimum practical interval between the adjacent pixels of the range image is $2.5 \mathrm{~mm}$. The corresponding image resolution is $128 \times 64$ pixels, which cannot meet the requirement of the analysis. So it is necessary to interpolate between the adjacent pixels to improve the data precision. The bicubic spline interpolation is used to improve the image resolution to $512 \times 256$ pixels, and the range image is named as $f(x, y)$. Fig $1(a)$ is the original collected range image. Fig 1(b) is the range image after the processing of noise moving and interpolating.

Suppose the footprint surface is twice differentiable. Because the footprint image data is sampled on a regular grid. The five partial derivatives $\left(f x, f_{y}, f_{x x}, f_{x y}\right.$ and $\left.f_{y y}\right)$ can be estimated respectively by fitting the local patch in a square window with the discrete orthogonal bi-quadratic polynomial [10]. The principal curvatures $\left(k_{1}\right.$ and $\left.k_{2}\right)$ and their directions $\left(\boldsymbol{e}_{1}\right.$ and $\left.\boldsymbol{e}_{2}\right)$ can be calculated based on the above five partial derivatives. The mean $H=\frac{1}{2}\left(k_{1}+k_{2}\right)$ is called the mean curvature. The product $K=k_{1} k_{2}$ is called the Gaussian curvature.

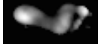

(a)

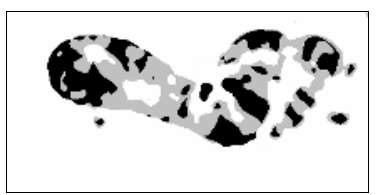

(d)

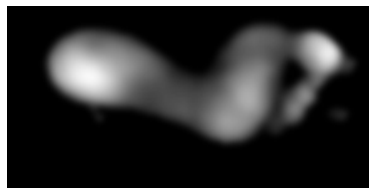

(b)

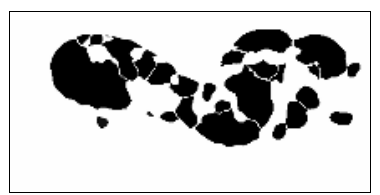

(e)

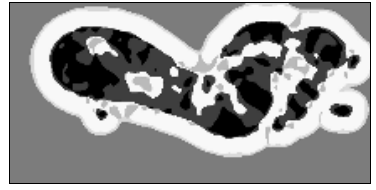

(c)

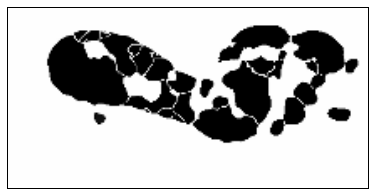

(f)

Fig. 1. Some footprint range image (a) range image (b) interpolated range image (c) curvature sign labeling (d) pre-segmentation result (e) result after the first growing (f) iterative growing segmentation result

According to the expert knowledge, the biometric features of bare footprint are mostly expressed by some interesting patches lying on toes, front sole, arch and heel. These interesting patches are generally peaks or ridges with the mean curvatures 
$H(i, j)<\varepsilon_{H}$. Based on the degree of containing biometric characteristics, we classify the regions of sign image into three categories and showed in Fig. 1(d):

$$
\begin{aligned}
& I=\left\{(i, j) \mid H(i, j)<\varepsilon_{H} \text { and } K(i, j)>\varepsilon_{K}\right\} \\
& I I=\left\{(i, j) \mid H(i, j)<\varepsilon_{H} \text { and } K(i, j) \leq \varepsilon_{K}\right\} \\
& I I I=\left\{(i, j) \mid H(i, j) \geq \varepsilon_{H}\right\}
\end{aligned}
$$

where $\varepsilon_{H}(>0)$ and $\varepsilon_{K}(>0)$ are two preset zero thresholds. Fig. 1(c) shows the curvature sign image of the bear footprint according literature [10].

\subsection{Region Growing}

In the pre-segmentation result, the positions of the interesting patches which containing most biometric characteristics can be located with Type $I$ areas. In this step, we take the regions of Type $I$ as seeds to track the boundaries of each interesting patches. Type $I I$ areas will be disintegrated and absorbed into the interesting patches.

Suppose that footprint range image is a twice-differentiable surface. The principal curvatures and directions are continuous. Let the principal curvatures of pixel $(i, j)$ are $k_{1}(i, j)$ and $k_{2}(i, j)$. Without loss of generality, we assume $k_{1}<k_{2}$. The definition of Type $I$ areas means $k_{1}+k_{2}<0$ and $k_{1} k_{2}>0$. So it can be deduced that $k_{1}$ and $k_{2}$ are both negative for type $I$ areas. For Type $I I$ areas, $k_{1}+k_{2}<0$ and $k_{1} k_{2} \leq 0$, which means that $k_{1}$ is negative and $k_{2}$ is positive. Fig. 2(a) shows the zoomed-in local surface of the middle toe of the footprint in Fig. 1. Fig. 2(b) and Fig. 2(c) give respectively the intensities and directions ( $\boldsymbol{e}_{2}$ projected to the zero-range plane) of its principal curvature $k_{2}$. We can see that at the boundaries of this interesting patch, the principal directions of $k_{2}$ are approximatively orthogonal to the tangent of the boundary curve, and the intensities of $k_{2}$ reach local maxima at their directions.

We design a region growing method based on the curvature characteristics to search the boundary of the interesting patches:

1. Seed regions. Take the separate regions of Type $I$ as seed regions, and sign them as $R_{1}, R_{2}, \ldots, R_{n}$.

2. Growing Principle. For any neighbor point $P$ of region $R_{i}(i=0,1, \ldots, n)$, we examine two adjacent points in the direction of $k_{2}$ (no consider the sign of the direction). The two adjacent points lie respectively inside and outside $R_{\mathrm{i}}$, denoted respectively as $P_{\text {in }}$ and $P_{\text {out }}$. We embody $P$ into $R_{i}$ if it meets all the following three conditions: (i) $P$ is the point of Type $I I$ areas; (ii) $k_{2}\left(P_{\text {in }}\right)<k_{2}(P)$; (iii) $P_{\text {out }}$ is not the point of Type $I I$, or if $P_{\text {out }}$ is in Type $I I$ it must be satisfied that $k_{2}(P)<k_{2}\left(P_{\text {out }}\right)$.

The above growing conditions lead the boundary of the interesting patches to grow and cease at the local maxima of $k_{2}$. But after growing there are still some points in Type II not included by any interesting patch (see Fig. 1(e)). For these residual areas, we take the small areas as noise and mark them as background. The residual areas of larger size are taken as the new interesting patches, and the same growing procedure are performed on them by taking the least mean curvature points of each area as the seeds. The growing procedure is iteratively applied until all the points of type $I I$ areas are classified into the interesting patches or marked as background. 


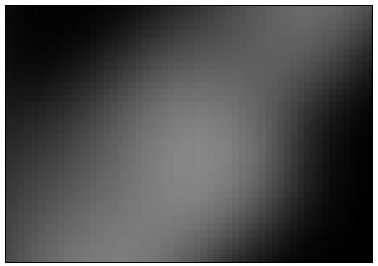

(a)

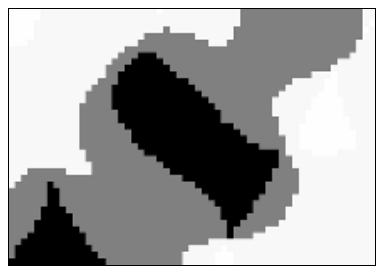

(d)

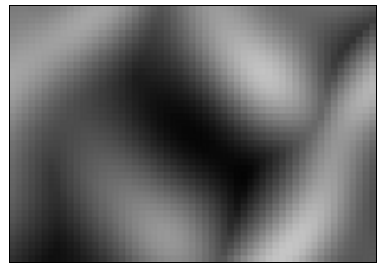

(b)

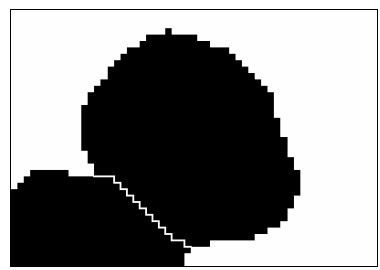

(e)

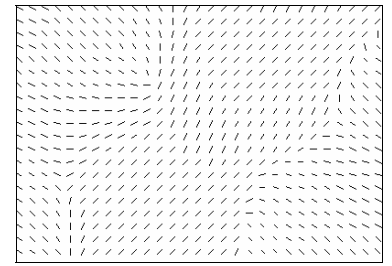

(c)

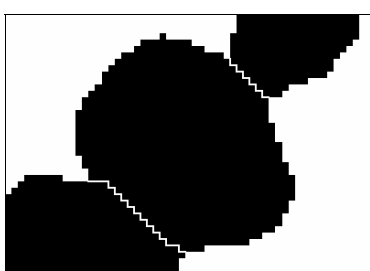

(f)

Fig. 2. Local patch round the middle toe (a) range image (b) the bigger principal curvature k2 (c) projection form the principal direction of $\mathrm{k} 2$ to the xoy plane (d) pre-segmentation result (e) result after growing once (f) result after iterative growing

In Fig. 1 and Fig. 2, we mark the interesting patches with black areas and the borderlines between two adjacent patches are drawn manually with white curves for clearly demarcated. The segmentation result of Fig. 2 shows that even if there is no peak in the index toe part, the interesting patch of index toe can still be found.

\subsection{Multi-scale Fusion}

The difficulties of computerized analysis of footprint are mainly caused by the instabilities of ground conditions and physical behavior. To lessen the disturbances of noises and instable factors, in section 2.1 we fit the footprint surface with different discrete orthogonal bi-quadratic polynomials which have different window sizes. Based on these fitting results with different window sizes, we can get different segmentation results. Then we fuse these results to get a more stable result.

Suppose that the footprint surface is fitted with a window of $l \times l$ size. With this fitting window we evaluate the curvatures, then after pre-segmentation and iteratively region growing the segmentation result of this size can be obtained. Let the bigger principal curvature is depicted with $k_{12}$ and the edge point set of the segmentation result is $S_{l}$. We define an edge strength image $E_{l}$ to represent the edge information of the footprint. For any pixel of $E_{l}$, we calculate the value of $g(d(i, j, m, n)) k_{l 2}(m, n)$ to measure the edge impact of the edge point $(m, n)$ on the pixel $(i, j)$, where $d(i, j, m, n)$ is the Euclidean distance between $(i, j)$ and $(m, n)$ and $g(\cdot)$ is a monotone descending function at the range of $[0,+\infty)$. Then we choose the maximum edge impact from all the edge points as the value of $E_{l}(i, j)$ :

$$
E_{l}(i, j)=\max _{(m, n) \in S_{l}} g(d(i, j, m, n)) k_{l 2}(m, n)
$$


With $t$ different window sizes $l_{1}, l_{2}, \ldots, l_{n}, t$ different edge strength images can be obtained for the same footprint. A fusion edge image is calculated by averaging the edge strength images of different sizes:

$$
E(i, j)=\frac{1}{t} \sum_{s=1}^{t} E_{l_{s}}(i, j)
$$

Watershed [13] is an utilizable tool to track precisely the boundaries of the fusion edge image. But some shivers can be created using this method. To avoid the oversegmentation, geodesic reconstruction technology [14] is applied on the fusion edge image before the watershed processing (see Fig. 3).

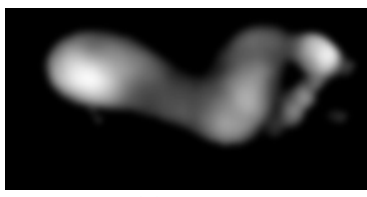

(a)

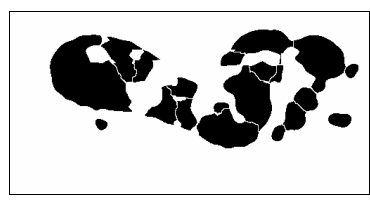

(d)

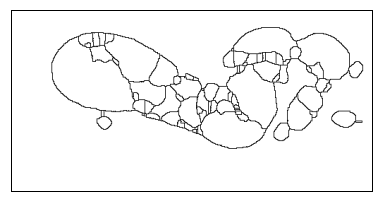

(g)

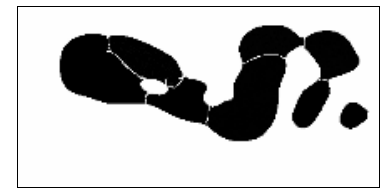

(b)

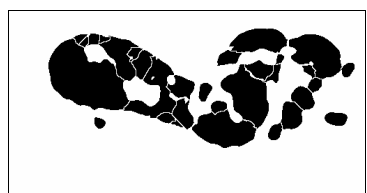

(e)

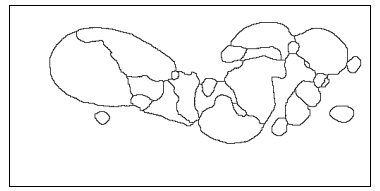

(h)

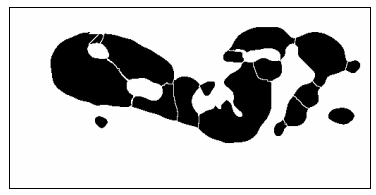

(c)

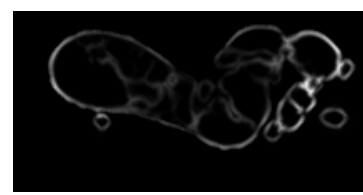

(f)

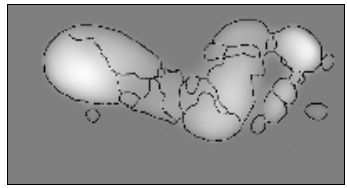

(i)

Fig. 3. Segmentation and fusion results (a) interposed range image (b)(c)(d)(e) segmentation results with $49 \times 49,25 \times 25,13 \times 13$ and $7 \times 7$ window operator (f) fusion image of five segmentation results above $(\mathrm{g})$ watershed segmentation result of the fusion image $(\mathrm{h})$ geodesic reconstruction and watershed segmentation result of the fusion image (i) the rang image covered with segmentation result

\section{Footprint Description}

As the complexity of the footprint shape and the multiplicity of the segmentation result, we use the man-machine interactive method to select five toes, heavy pressed patches, the foot arch area and the heel region from the segmentation results. It can guarantee the correctness of the characteristic regions, and is of advantage to merge over-segmentation regions.

After the segmentation, an effective and reasonable description to the footprint is another key to obtain the stable characteristic for further recognition. Although the footprint is easy to be interfered by ground conditions and personal behaviors, it contains the skeleton and muscle characteristic of each person. To effectively extract 
the biometric features of the footprint, we use different parameter surface to represent each regions of the footprint surface. According to the shape characteristic of each patch, we use superquadric fitting for five toes, heavy pressed patches and the heel region, and saddle (a hyperbolic paraboloid) fitting for the foot arch area.

Superquadrics are three-dimensional models suitable for part-level representation of objects, and it can be reconstructed from range images. A superquadric surface is defined by the following implicit equation

$$
\left(\left(\frac{x}{a_{1}}\right)^{2 / \varepsilon_{2}}+\left(\frac{y}{a_{2}}\right)^{2 / \varepsilon_{2}}\right)^{\varepsilon_{2} / \varepsilon_{1}}+\left(\frac{z}{a_{3}}\right)^{2 / \varepsilon_{1}}=1
$$

where $a_{1}, a_{2}$ and $a_{3}$ define the superquadric size, and $\varepsilon_{1}$ and $\varepsilon_{2}$ define a smoothly changing family of shapes from rounded to square. Saddles also can be reconstructed from range images. A saddle surface is defined by the following equation

$$
(a x)^{2}-(b y)^{2}=c z
$$

According to the shape of the foot arch, we set $c=1$.

To recover above surfaces in a general position, we should add six parameters: Euler angle $(\phi, \theta, \psi)$ define the orientation in space, and $p_{x}, p_{y}, p_{z}$ define the position in space. The superquadrics and saddles for general position is defined as following implicit equation:

$$
\begin{gathered}
F\left(x, y, z ; a_{1}, a_{2}, a_{3}, \varepsilon_{1}, \varepsilon_{2}, \phi, \theta, \psi, p_{x}, p_{y}, p_{z}\right)=0 \\
G\left(x, y, z ; a, b, \phi, \theta, \psi, p_{x}, p_{y}, p_{z}\right)=0
\end{gathered}
$$

For superquadric and saddle models are nonlinear, the Levenberg-Marquardt method allows one to iteratively find their parameters [15][16][17].

Fig. 4 shows the superquadric and saddle fitting results for the range image of Fig. 3(a). By surface fitting, we describe the foot shape characteristics with several groups of superquadric and saddle parameters, which can be taken as the feature parameters for foot recognition.

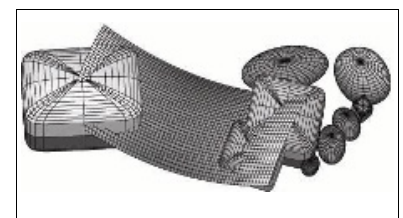

(a)

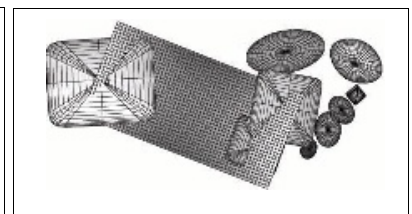

(b)

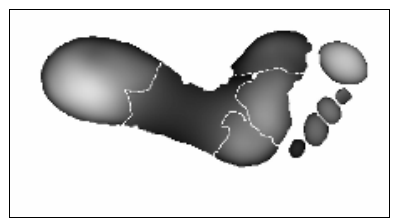

(c)

Fig. 4. Superquadrics and saddles fitting results of Fig. 3(a). (a)(b) the 3-dimensional fitted models of each regions from different viewpoints. (c) a result obtained by replacing the toe and foot arch regions with the fitted data. 


\section{Experiments and Conclusion}

The curvature sign based segmentation method is the most generally used in the segmentation of range surface. So we compare the result of our method with the curvature sign image. We test our method on a number of footprint range images and obtain excellent segmentation results. Fig 5 gives some instances of segmentation results of our method and the corresponding curvature sign images [10]. It can be seen that our method can track the boundaries correctly and the extracted interesting patches comprise significant biometric information which is the foundation of feature analysis and recognition.

The proposed segmentation method for stereo bare footprint has the following merits:

1. The feature parameters used in the segmentation method, including the principle curvatures and directions, contain the entire geometric characteristics of the surface. The characteristics used in the paper are invariable to the translation and rotation.

2. The growing principle can accurately track the boundaries of the interesting patches. For those interesting patches that have no peak, the region can also be detected and segmented. The under-segmentation, such as two or more toes being embodied in the same patch, is on the whole avoided.

3. The applications of multi-scale fusion, geodesic reconstruction and watershed extract the stable edge information efficiently and simultaneously remove most fake edges caused by noises.

4. The superquadric and saddle parameters describe the biometric characteristics and fade the influence of ground conditions and personal behaviors.

The experiment results show that the performance of our segmentation method is excellent. The segmentation results provide a secure platform for the future works on biometric feature extraction and recognition.

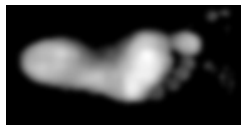

(a1)

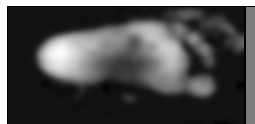

(b1)

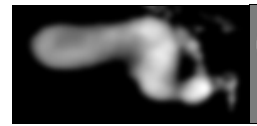

$(\mathrm{c} 1)$

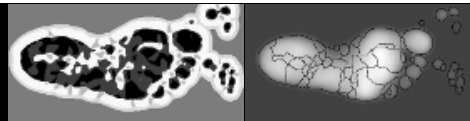

(a2)

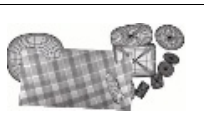

(a4)

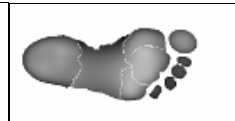

(a5)

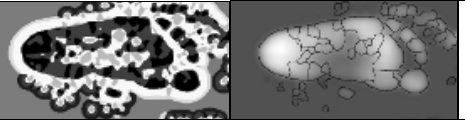

(b2)

(b3)

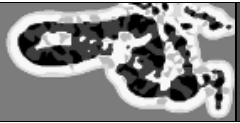

(c2)

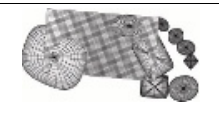

(b4)

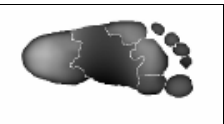

(b5)

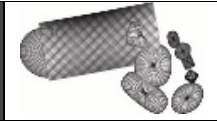

(c4)

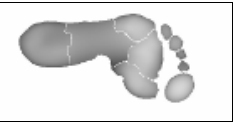

(c5)

Fig. 5. Experiments on footprint range images. (a1)(b1)(c1) the range images (a2)(b2)(c2) the corresponding curvature sign images $(\mathrm{a} 3)(\mathrm{b} 3)(\mathrm{c} 3)$ the edge images of the final segmentation results (a4)(b4)(c4) the 3-dimensional fitted models of each regions (a5)(b5)(c5) results obtained by replacing the toe and foot arch regions with the fitted data 


\section{Acknowledgements}

This research is supported by the National Natural Science Foundation of China under Grant No. 60272004.

\section{References}

1. Chirillo, John., Blaul, S.: Implementing Biometric Security. John Wiley \& Sons. (2003)

2. Wang, Q.J., Han, J.L., Zheng, D.C., Wu Z.L.: The Quantitative Inspection of Footprint and Gait. Chinese People's Public Security University Press (1992) (in Chinese)

3. Tian, Y., Ping X.J., Wang Y.J.: A New Method about 3D Surface Recognition. Proceedings of the 5th Joint Conference on Information Sciences. 1 (2000) A147-A150

4. Nakajima, K., Mizukami, Y., Tanaka, K., Tamura, T.: Footprint-Based Personal Recognition. IEEE Trans. on Biomedical Engineering. 47 (2000) 1534-1537.

5. Kennedy, R.B., Pressman, S., Chen, S., Petersen, P.H., Pressman, A.E.: Statistical Analysis of Barefoot Impressions. Journal of Forensic Sciences. 48 (2003) 55-63

6. Fan, T., Medioni, G., Nevatia, R.: Segmented description of 3-D surfaces. IEEE Journal of Robotics and Automation, , 3 (1987) 527-538

7. Bellon, O.R.P., Silva, L.: New Improvements to Range Image Segmentation by Edge Detection. IEEE Signal Processing Letters. 9 (2002) 43-45

8. Jiang, X.Y., Bunke, H.: Edge Detection in Range Images Based on Scan Line Approximation. Computer Vision and Image Understanding. 73 (1999) 183-199

9. Zhu, S.C., Yuille, A.: Region Competition: Unifying Snakes, Region Growing, and Bayes/ MDL for Multi-band Image Segmentation. IEEE Trans on PAMI. 18 (1996) 884-900

10. Besl, P.J., Jain, R.C.: Segmentation Through Variable Order Surface Fitting. IEEE Trans. on PAMI. 10 (1988) 167-192

11. Koster, K., Spann, M.: MIR: An Approach to Robust Clustering Application to Range Image Segmentation. IEEE Trans. on PAMI. 22 (2000) 430-444

12. Yokoya, N., Levine, D.: Range Image Segmentation Based on Differential Geometry: A Hybrid Approach. IEEE Trans. on PAMI. 11 (1989) 634-649

13. Vincent, L., Soille, P.: Watersheds in Digital Spaces: An Efficient Algorithm Based on Immersion Simulations. IEEE Trans. on PAMI. 13 (1991) 583-598

14. Vincent, L.: Morphological Grayscale Reconstruction in Image Analysis: Applications and Efficient Algorithms. IEEE Trans. on Image Processing. 2 (1993) 176-201

15. Leonardis, A., Jaklic, A., Solina, F.: Superquadrics for Segmenting and Modeling Range Data. IEEE Trans. on PAMI. 19 (1997) 1289-1295

16. Whaite, P., Ferrie, F. P.: From Uncertainty to Visual Exploration. IEEE Trans. on PAMI. 13 (1991) 1038-1049

17. Press, W. H., Teukolsky, S. A., Vetterling, W. T., Flannery, B. P.: Numerical Recipes in C: The Art of Scientific Computing. London: Cambridge University Press, 1992 